\title{
Recent results from the SND detector
}

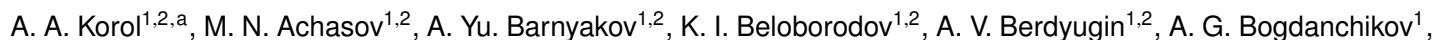
A. A. Botov ${ }^{1}$, T. V. Dimova ${ }^{1,2}$, V. P. Druzhinin ${ }^{1,2}$, V. B. Golubev ${ }^{1,2}$, L. V. Kardapoltsev ${ }^{1,2}$, A. G. Kharlamov ${ }^{1,2}$, S. V. Koshuba ${ }^{1}$, D. P. Kovrizhin ${ }^{1,2}$, A. S. Kupich ${ }^{1}$, K. A. Martin ${ }^{1,2}$, A. E. Obrazovsky ${ }^{1}$, E. V. Pakhtusova1, S. I. Serednyakov ${ }^{1,2}$, D. A. Shtol ${ }^{1,2}$, Z. K. Silagadze ${ }^{1,2}$, I. K. Surin ${ }^{1,2}$, Yu. V. Usov ${ }^{1,2}$, and A. V. Vasiljev ${ }^{1,2}$ The SND Collaboration

${ }^{1}$ Budker Institute of Nuclear Physics, Novosibirsk, 630090, Russia

${ }^{2}$ Novosibirsk State University, Novosibirsk, 630090, Russia

\begin{abstract}
Recent results of the SND detector experiments are presented. Data were taken at the VEPP2000 and the VEPP-2M $e^{+} e^{-}$colliders in the Novosibirsk in the c.m. energy ranges from 0.3 to 2.00 $\mathrm{GeV}$. Integrated luminosity used are 70pb-1 and 25pb-1 respectively. The cross sections of the $e^{+} e^{-} \rightarrow$ $\pi^{0} \gamma, K^{+} K^{-}, \omega \pi^{0}, \pi^{+} \pi^{-} \pi^{0} \eta, \omega \eta, \omega \eta \pi^{0}$ processes has been measured.
\end{abstract}

\section{Detector and experiment}

We present the results of study the data collected on the SND detector [1-3] at the electron-positron colliders VEPP-2M [4] and VEPP-2000 [5] in the center of mass energy range $0.3 \div 2.0 \mathrm{GeV}$. The SND detector consists of several subsystems (Fig.1) including a spherical electromagnetic calorimeter, a cylindrical tracking system, a threshold Cherenkov counters and a muon detector. Electromagnetic calorimeter covers 0.95 of the full solid angle, has thickness $13.4 \mathrm{X} 0$, energy resolution $\delta E / E=$ $0.042 / \sqrt[4]{E(G e V)}$, and angle resulution $\delta \varphi=1.5^{\circ}$. Tracking system covers 0.94 of the full solid angle, has angle resolution $\delta \varphi=0.45^{\circ}, \delta \theta=0.8^{\circ}$.

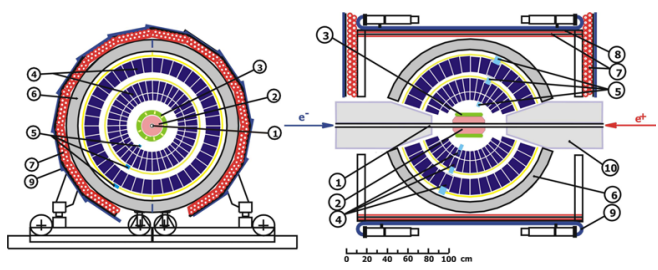

Figure 1. SND detector layout: 1 - beam pipe, 2 - tracking system, 3 - aerogel Cherenkov counter , 4 - NaI(Tl) crystals, 5 phototriodes, 6 - iron muon absorber, 7-9- muon detector, 10 focusing solenoids .

The integrated luminosity for experiments with SND detector at both the VEPP-2M and VEPP2000 $e^{+} e^{-}$colliders is summarized in the Table.1.

During 2010-2013 the luminosity was limited by the shortage of positrons. This problem is to be solved in the

\footnotetext{
a e-mail: a.a.korol@inp.nsk.su
}

Table 1. Used data

\begin{tabular}{|c|c|c|c|}
\hline \multicolumn{4}{|c|}{ VEPP-2M } \\
\hline \hline & Below $\phi$ & Around $\phi$ & Above $\phi$ \\
\hline \hline$I L, p b^{-1}$ & 9.1 & 13.2 & 8.8 \\
\hline$\sqrt{s}, \mathrm{GeV}$ & $0.36-0.97$ & $0.98-1.06$ & $1.06-1.38$ \\
\hline \hline \multicolumn{4}{|c|}{ VEPP-2000 } \\
\hline \hline$I L, p b^{-1}$ & 15.5 & 6.9 & 47.0 \\
\hline$\sqrt{s}, \mathrm{GeV}$ & $0.30-0.97$ & $0.96-1.05$ & $1.05-2.00$ \\
\hline
\end{tabular}

ongoing upgrade of the VEPP-2000 complex with electrons and positrons transported from the VEPP-5 injection complex. Experiments at upgraded VEPP-2000 are expected to start by the end of 2016.

\section{Known processes cross section measurement}

The $e^{+} e^{-} \rightarrow \pi^{0} \gamma$ process cross section was measured [6] using full statistics collected at VEPP-2M and VEPP-2000 colliders. This process has the third largest cross section below $1 \mathrm{GeV}$, its study allows to obtain parameters of electromagnetic radiative decays of the vector mesons $(\rho, \omega, \phi)$, and allows to extract information on the transition form factor $\pi^{0} \gamma \gamma^{*}$. The process $e^{+} e^{-} \rightarrow 2 \gamma$ is used for normalization. Common selection criteria for $2 \gamma$ and $3 \gamma$ final states are trigger, charged tracks absense, large total energy deposition, low momentum estimated from calorimeter, and muon system veto. Final selection is based on 4C kinematic fit: $\chi_{3 \gamma}^{2}<30,36^{\circ}<\theta_{\gamma}<144^{\circ}$, $80<M_{r c}<190 \mathrm{MeV}$. Here $M_{r c}$ is the mass recoiling against largest energy photon. The number of signal events is determined from the fit of $\pi^{0}$ in the $M_{r c}$ spectrum. 
This is the most precise measurement of the $e^{+} e^{-} \rightarrow$ $\pi^{0} \gamma$ process cross section (Fig.2). Systematic uncertainty at the $\omega$ peak is $1.4 \%$, main contributions from luminosity $(1.2 \%)$ and selection criteria $(0.6 \%)$.

Using PDG [7] value for $B\left(\omega \rightarrow \pi^{+} \pi^{-} \pi^{0}\right) \cdot B(\omega \rightarrow$ $\left.e^{+} e^{-}\right)$we have obtained $\Gamma\left(\omega \rightarrow \pi^{0} \gamma\right) / \Gamma\left(\omega \rightarrow \pi^{+} \pi^{-} \pi^{0}\right)=$ $0.0992 \pm 0.0023$, which is higher than the KLOE [8] value $0.0897 \pm 0.0016$ by $3.4 \sigma$. Measured $B\left(\rho \rightarrow \pi^{0} \gamma\right)=(4.20 \pm$ $0.47 \pm 0.22) \cdot 10^{-4}$ is by $1.8 \sigma$ lower than the current PDG value $(6.0 \pm 0.8) \cdot 10^{-4}$, but agrees with the branching fraction for the charged mode $B\left(\rho^{ \pm} \rightarrow \pi^{ \pm} \gamma\right)=(4.5 \pm 0.5) \cdot 10^{-4}$. For $\phi$-meson decay we get $B\left(\phi \rightarrow \pi^{0} \gamma\right) \cdot B\left(\phi \rightarrow e^{+} e^{-}\right)=$ $\left(3.92_{-0.40}^{+0.71} \pm 0.51\right) \cdot 10^{-7}$.
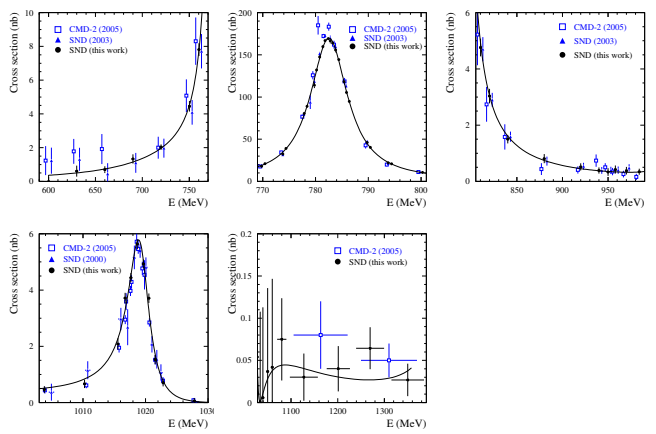

Figure 2. $e^{+} e^{-} \rightarrow \pi^{0} \gamma$ cross section

We studied the $e^{+} e^{-} \rightarrow K^{+} K^{-}$process [9]. Preliminary events selection is trigger and 2 back-to-back central tracks in drift chamber. Then for events with absense of the signal from threshold Cherenkov counter for at least one track the number of events is fitted from normalized energy deposition distribution. Our cross section measurement agrees with the BABAR data and has comparable or better accuracy (Fig.3).

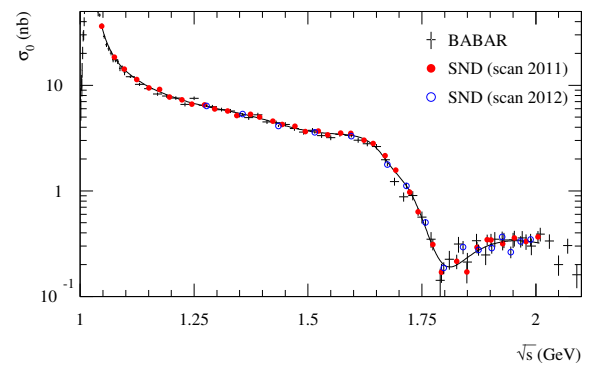

Figure 3. $e^{+} e^{-} \rightarrow K^{+} K^{-}$cross section

The process $e^{+} e^{-} \rightarrow \omega \rightarrow \pi^{0} \pi^{0} \gamma$ was earlier studied in [10] but that study has a mistake in the radiative correction calculation. This is fixed now ([11]) and also additional data are taken into account $\left(37 p b^{-1}\right.$ instead of $\left.25 p b^{-1}\right)$. The result shown at Fig.4.
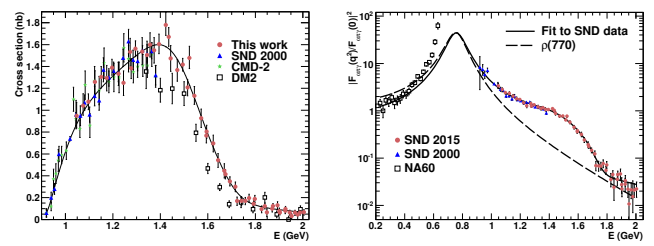

Figure 4. $e^{+} e^{-} \rightarrow \omega \rightarrow \pi^{0} \pi^{0} \gamma$ cross section and $\omega \pi^{0} \gamma$ form factor

\section{New processes study}

Below $2 \mathrm{GeV}$ the total hadronic cross section is calculated as a sum of exclusive cross sections. Currenly the exclusive and inclusive data below $2 \mathrm{GeV}$ are in reasonable agreement Fig.[12]. But in the energy region 1.5-2.0 GeV exclusive data are incomplete. There are no experimental data on the final states $\pi^{+} \pi^{-} \pi^{0} \eta, \pi^{+} \pi^{-} \eta \eta, \pi^{+} \pi^{-} \pi^{0} \pi^{0} \pi^{0}, \pi^{+} \pi^{-} \pi^{0} \pi^{0} \eta, \ldots$, so their study is very important.

We studied the $e^{+} e^{-} \rightarrow \pi^{+} \pi^{-} \pi^{0} \eta$ process [13]. This is the first measurement of its cross-section (Fig.5) and intermediate states. The intermediate states found are

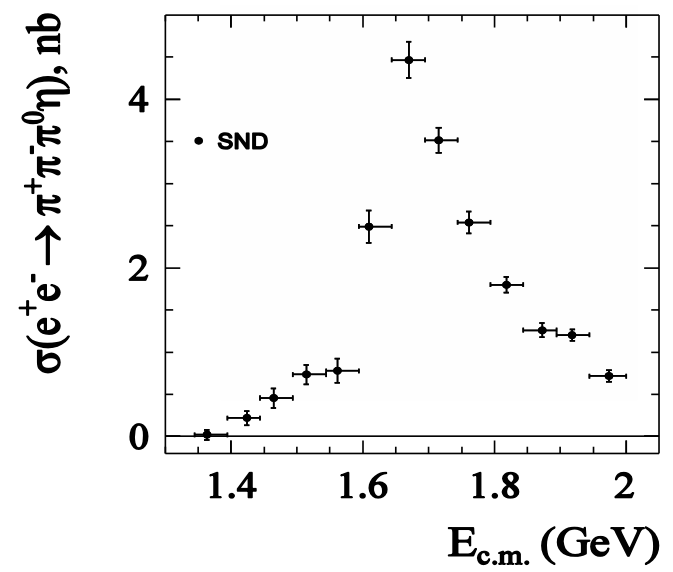

Figure 5. Process $e^{+} e^{-} \rightarrow \pi^{+} \pi^{-} \pi^{0} \eta$ cross section

$\omega \eta, \phi \eta$, structureless $e^{+} e^{-} \rightarrow \pi^{+} \pi^{-} \pi^{0} \eta$, and $a_{0}(980) \rho$. Above $1.8 \mathrm{GeV}$ the last one is the dominant reaction mechanism (Fig.6). The process $e^{+} e^{-} \rightarrow \omega \eta$ has been measured separately (Fig.7). There is a significant difference between SND result and the previous BABAR measurement [14].

There is also the first study of the process $e^{+} e^{-} \rightarrow$ $\omega \pi^{0} \eta \rightarrow 7 \gamma$ [15]. Events of the $e^{+} e^{-} \rightarrow \pi^{0} \pi^{0} \eta \gamma \rightarrow 7 \gamma$ final state are selected. The dominant intermediate state is $\omega \pi^{0} \eta$. No noticeable $\eta^{\prime} \gamma$ signal is observed. The $\pi^{0} \eta$ mass spectrum for selected $\omega \pi^{0} \eta$ events is well described by the model of the $a^{0}(980) \omega$ intermediate state (Fig.8).

The cross section energy dependence is described by a single-resonance model. The resonance mass and width 

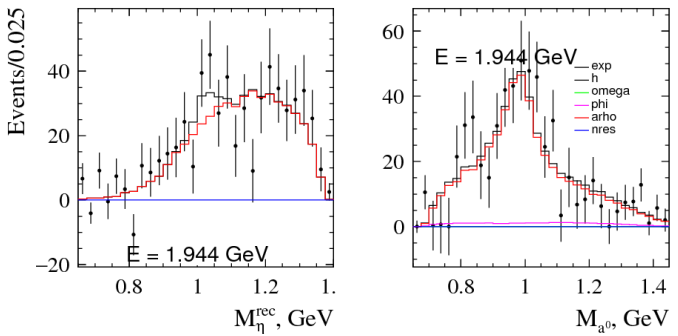

Figure 6. Mass recoling against $\eta$ and $\eta \pi^{0}$ invariant mass.

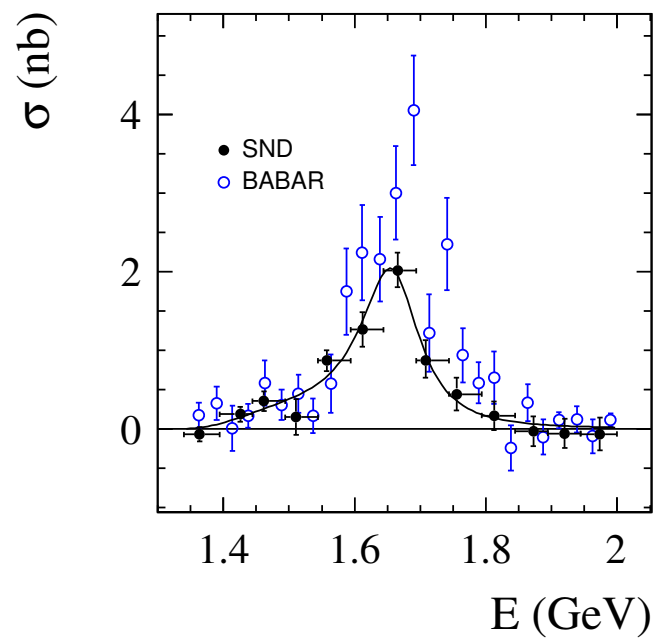

Figure 7. Process $e^{+} e^{-} \rightarrow \omega \eta$ cross section
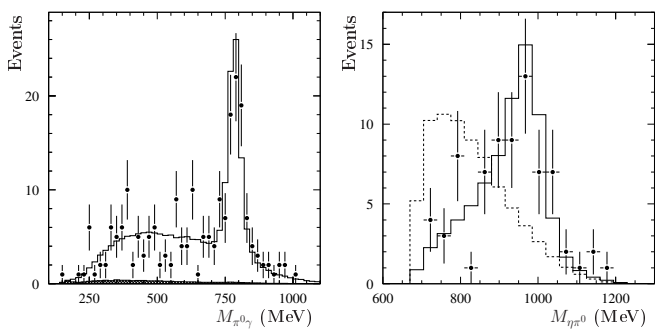

Figure 8. $\pi^{0} \gamma$ mass, $\eta \pi^{0}$ mass

are consistent with those for $\rho(1700)$ better than nonresonant at $1.2 \sigma$ level (Fig.9).

\section{Conclusions}

During 2010 - 2013 the SND detector accumulated $\sim 70 \mathrm{pb}^{-1}$ of integrated luminosity at the VEPP-2000 electron-positron collider in the c.m. energy range $0.3-$ $2 \mathrm{GeV}$. Data analysis on hadron production is in progress. The obtained results have comparable or better accuracy than previous measurements $\left(\pi^{0} \gamma, K^{+} K^{-}, \omega \pi^{0}\right)$. For some

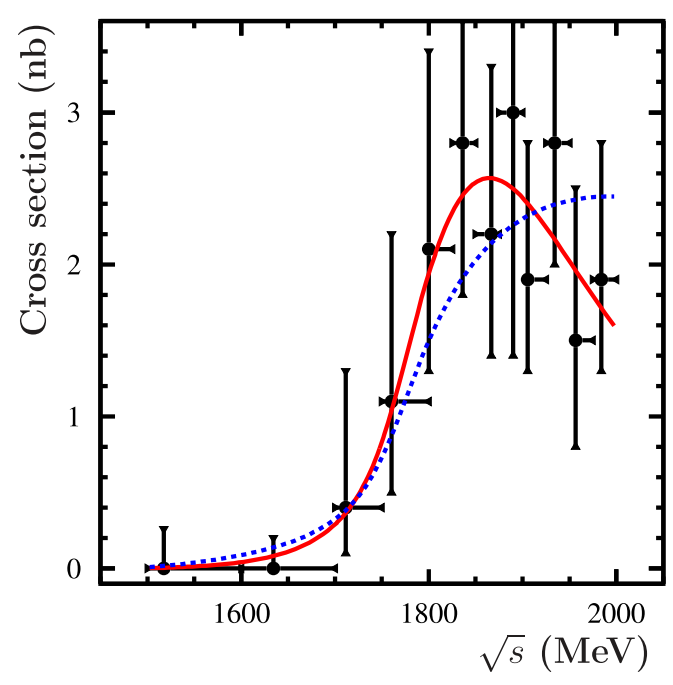

Figure 9. Process $e^{+} e^{-} \rightarrow \omega \pi^{0} \eta$ cross section

processes the cross sections have been measured for the first time $\left(\pi^{+} \pi^{-} \pi^{0} \eta, \omega \pi^{0} \eta\right) . a^{0}(980) \omega$ and $a^{0}(980) \rho$ intermediate states found to be significant in these processes. After VEPP-2000 upgrade the data taking runs will be continued with a goal of $\sim 1 \mathrm{fb}^{-1}$ of integrated luminosity.

\section{References}

[1] M.N. Achasov et al., Nucl. Instrum. Meth. A449, 125 (2000), hep-ex/9909015

[2] G.N. Abramov et al., eConf C010430, T10 (2001), [,122(2001)], hep-ex/0105093

[3] V.M. Aulchenko et al., Nucl. Instrum. Meth. A598, 102 (2009)

[4] I.A. Koop et al., Status of VEPP-2M collider, its upgrade plans and Novosibirsk Phi-factory project, in Physics and detectors for DAPHNE. Proceedings, 3rd International Workshop, DAPHNE'99, Frascati, Italy, November 16-19, 1999 (1999), pp. 393-404

[5] B.I. Khazin (CMD-3, SND), Nucl. Instrum. Meth. A623, 353 (2010)

[6] M.N. Achasov et al. (SND), Phys. Rev. D93, 092001 (2016), 1601.08061

[7] K.A. Olive et al. (Particle Data Group), Chin. Phys. C38, 090001 (2014)

[8] F. Ambrosino et al. (KLOE), Phys. Lett. B669, 223 (2008), 0807.4909

[9] M.N. Achasov et al., Phys. Rev. D94, 112006 (2016), 1608.08757

[10] M.N. Achasov et al., Phys. Rev. D88, 054013 (2013), 1303.5198

[11] M.N. Achasov et al., Phys. Rev. D94, 112001 (2016), 1610.00235

[12] F. Jegerlehner, EPJ Web Conf. 118, 01016 (2016), 1511.04473 
[13] M.N. Achasov et al., Phys. Rev. D94, 092002 (2016), 1607.00371

[14] B. Aubert et al. (BaBar), Phys. Rev. D73, 052003 (2006), hep-ex/0602006
[15] M.N. Achasov et al., Phys. Rev. D94, 032010 (2016), 1606.06481 